Disclosure of Interests: None declared

DOI: 10.1136/annrheumdis-2021-eular.3659

\section{POS0250 SIGNIFICANT DAMAGE OCCURS EARLY IN THE COURSE OF EOSINOPHILIC GRANULOMATOSIS WITH POLYANGIITIS AND IS MAINLY DUE TO DISEASE- RELATED SEQUELAE}

P. Delvino $^{1,2}$, A. Milanesi ${ }^{1}$, F. Brandolino ${ }^{1}$, S. Monti ${ }^{1,2}$, C. Montecucco ${ }^{1}{ }^{1} / R C C S$ Policlinico S. Matteo Foundation, University of Pavia, Rheumatology, Pavia, Italy; ${ }^{2}$ University of Pavia, PhD in Experimental Medicine, Pavia, Italy

Background: Following the introduction of effective immunosuppressive treatments, ANCA-associated vasculitides (AAV) have become chronic diseases with a remitting-relapsing course. Therefore, preventing chronic damage accrual during follow-up is critical, as relapses, treatment-related side effects, and comorbidities may significantly affect the long-term outcomes of AAV patients. At present, no study specifically evaluated the burden of damage in patients with eosinophilic granulomatosis with polyangiitis (EGPA).

Objectives: To describe short-term (6 months) and long-term (5 years) damage accrual in patients with newly diagnosed EGPA.

Methods: Patients diagnosed with EGPA, according to ACR criteria and/or Chapel Hill definitions and regularly followed-up in our vasculitis center for $\geq 5$ years were included. Damage accrual was assessed with the Vasculitis Damage Index (VDI). Short-term and long-term damage accrual was defined by VDI at 6 months and at 5 years, respectively, and categorized as related to vasculitis or its treatment. Results: VDI data at 6 months were available for 45 EGPA patients: 24 (53.3\%) female, mean age at diagnosis $51.6 \pm 13.0$ years. ANCA were positive in 17 patients $(37.8 \%)$, with MPO being the only detected enzyme immunoassay (EIA)-specificity. At 6 months mean VDI was $2.8 \pm 1.3 ; 25 / 45(55.6 \%)$ and $6 / 45$ patients $(13.3 \%)$ presented $\geq 3$ and $\geq 5$ items, respectively, whilst only 1 patient $(2.2 \%)$ showed no items of damage. VDI data at 5 years were available for $32 / 45$ EGPA patients $(71.1 \%)$ : $16(50 \%)$ female, mean age at diagnosis $51.5 \pm 13.1$ years. MPO-ANCA were positive in 13 patients (40.6\%). At 5 years mean VDI was $3.5 \pm 1.3$, with $26 / 32(81.3 \%)$ and $7 / 32$ patients $(21.9 \%)$ presenting $\geq 3$ and $\geq 5$ items, respectively; notably, no patients presented a VDl=0 at 5 years. The most frequent disease-related VDI items at 6 months and at 5 years were asthma, chronic sinusitis, peripheral neuropathy, cardiomyopathy, pulmonary function tests abnormalities and nasal blockage (Figure 1). Osteoporotic fractures, diabetes and systemic hypertension were the most commonly reported treatment-related items at 6 months and at 5 years (Figure 1). Damage accrual progressively rose during the 5 -year follow-up $(P=0.023)$, mainly due to disease-related items rather than treatment-related items both at 6 months (disease related VDI $2.6 \pm 1.2$, treatment-related VDI $0.3 \pm 0.6$ ) and at 5 years (disease related VDI 2.9 \pm 1.2 , treatment-related VDI $0.6 \pm 0,7)$. No significant difference in terms of damage accrual was observed between ANCA-positive and ANCA-negative patients $(P>0.5)$.

Conclusion: In our cohort of EGPA patients damage accrual occurs early, with more than half of the patients displaying $\geq 3$ VDI items already at 6 months. Poor control of previous disease activity, particularly ENT and respiratory manifestations, contributes to progressive damage accrual more than treatment side effects.

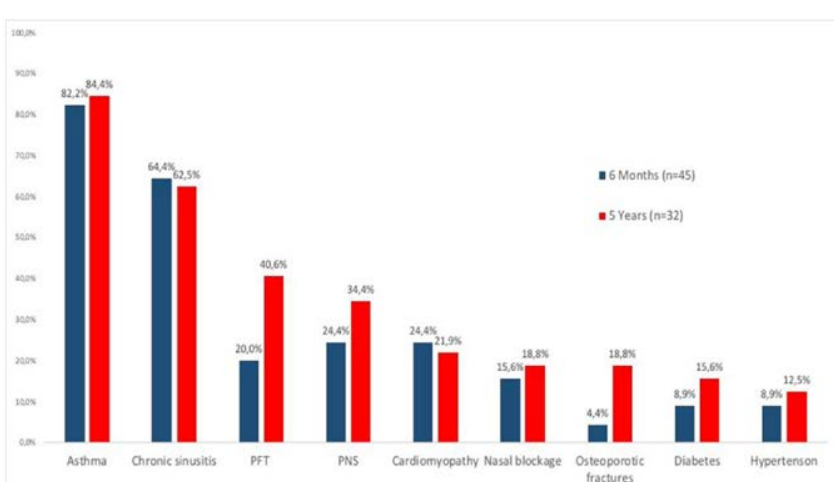

Figure 1. Disease-related and treatment-related VDI items at 6 months and at 5 years in patients with EGPA.

Disclosure of Interests: None declared

DOI: 10.1136/annrheumdis-2021-eular.2683

\section{POS0251 ARE CLINICAL FEATURES AND OUTCOMES OF ANCA (+) EGPA PATIENTS DIFFERENT FROM ANCA NEGATIVE ONES?}

E. Duran ${ }^{1}$, E. Bilgin ${ }^{1}$, E. Erkantarcı Levent ${ }^{2}$, Ö. Can Bostan ${ }^{3}$, L. Kılıç ${ }^{1}$, E. Damadoğlu ${ }^{3}, \mathrm{~S}$. Kiraz ${ }^{1}$, O. Karadag ${ }^{1,4} .{ }^{1}$ Hacettepe University School of
Medicine, Department of Internal Medicine, Division of Rheumatology, Ankara, Turkey; ${ }^{2}$ Hacettepe University School of Medicine, Department of Internal Medicine, Ankara, Turkey; ${ }^{3}$ Hacettepe University School of Medicine, Department of Chest Disease, Division of Allergy and Clinical Immunology, Ankara, Turkey; ${ }^{4}$ Hacettepe University School of Medicine, Vasculitis Research Centre, Ankara, Turkey

Background: Recent reports describe EGPA as having two subgroups as ANCA positive and ANCA negative. Furthermore, there could be differences in terms of clinical features and outcomes (1).

Objectives: The aim of this study was to compare the clinical characteristics and outcomes of ANCA positive EGPA patients with those of ANCA negative ones. Methods: This retrospective, descriptive study included 50 EGPA patients (male/ female:13/27) from our prospective vasculitis database from October 2014 (2). The patients had fulfilled the ACR 1990 or DCVAS criteria for EGPA. In addition to clinical features (activity index) and treatment regimens, outcomes of patients (relapse and damage) were also reviewed. For relapse-free survival analysis, time to first relapse was compared according to ANCA phenotype by KaplanMeier survival analysis.

Results: Of the patients, 17 (34\%) were in ANCA (+) group, $33(66 \%)$ were in ANCA (-) group. Renal involvement and peripheral neuropathy were more frequent in ANCA (+) patients whereas ANCA (-) patients were significantly younger at the time of diagnosis and they had more nasal polyposis. The median BVAS at the EGPA diagnosis was significantly high in ANCA (+) group. All 3 of patients with cardiac involvement were in the ANCA (-) group but difference was found in terms of FFS and VDI between ANA (+) and (-) groups. However, pulse steroid and cyclophosphamide treatments were more commonly used in ANCA $(+)$ group while mepolizumab were used in $1 / 4$ of ANCA (-) patients (Table 1). During median 47 (IQR 69.9) months follow up, about $40 \%$ of patients had at least one relapse but there was no difference for relapse-free survival rate according to the ANCA status (Figure 1).

Conclusion: Not only clinical features and disease activity but also treatments received were significantly different between ANCA (+) and (-) patients. These results could partially define two distinct subgroups of EGPA. However, these groups were similar regarding damage and relapse.

\section{REFERENCES:}

[1] Comarmond C, Pagnoux C, Khellaf M, Cordier JF, Hamidou M, Viallard JF, et al. Eosinophilic granulomatosis with polyangiitis (Churg-Strauss): Clinical characteristics and long-term followup of the 383 patients enrolled in the French Vasculitis Study Group cohort. Arthritis and rheumatism. 2013;65(1):270-81.

[2] Karadağ Ö, Bilgen SA, Armagan B, Sari A, Erden A, Batu E D et al. Twoyear Results of a Prospective Vasculitis Cohort from Eastern Mediterranean: Demographic Characteristics and Distribution of the Vasculitides Frequencies. Rheumatology, 2017; 56(suppl_3), iii88-iii95

Table 1. Demographics and clinical characteristics of the patients with EGPA during disease course

\begin{tabular}{|c|c|c|c|}
\hline Characteristics $^{*}$ & $\begin{array}{c}\text { ANCA }(+) \\
(n=17)\end{array}$ & $\begin{array}{c}\text { ANCA (-) } \\
(n=33)\end{array}$ & $P$ \\
\hline Age at the diagnosis (years) & $53.8 \pm 16.3$ & $37.9 \pm 14.3$ & 0.001 \\
\hline Sex, female & $10(58.8)$ & $17(51.5)$ & 0.62 \\
\hline Asthma & $12(70.6)$ & $29(87.9)$ & 0.13 \\
\hline Nazal polyp & $2(11.8)$ & $15(45.5)$ & 0.017 \\
\hline 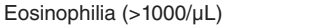 & $11(64.7)$ & $27(81.8)$ & 0.18 \\
\hline Disease duration (years) & $4.1(5.9)$ & $3.7(5.9)$ & 0.69 \\
\hline \multicolumn{4}{|l|}{ Organ specific involvement, \% } \\
\hline - Pulmonary & 76.5 & 78.8 & 0.85 \\
\hline - ENT & 82.4 & 87.9 & 0.59 \\
\hline - Renal & 52.9 & 3 & $<0.001$ \\
\hline - PNS & 47.1 & 9.1 & 0.002 \\
\hline - Cardiac & 0 & 9.1 & $\mathrm{~N} / \mathrm{A}$ \\
\hline BVAS at the diagnosis & $17(13)$ & $9(4)$ & 0.002 \\
\hline \multicolumn{4}{|l|}{ Revized FFS } \\
\hline-0 & $11(64.7)$ & $26(78.8)$ & 0.28 \\
\hline$-\geq 1$ & $6(35.3)$ & 7 (78.8) & \\
\hline VDI & $1(1)$ & $1(1)$ & 0.41 \\
\hline \multicolumn{4}{|l|}{ Treatment regimens for induction, \% } \\
\hline - Pulse steroid & 64.7 & 11.2 & 0.002 \\
\hline - Cyclophosphamide & 58.8 & 18.2 & 0.004 \\
\hline - Rituximab & 11.8 & 3 & 0.21 \\
\hline - Mepolizumab & 0 & 12.1 & $\mathrm{~N} / \mathrm{A}$ \\
\hline \multicolumn{4}{|c|}{ Treatment regimens for maintenance, $\%$} \\
\hline - Rituximab & 11.8 & 3 & 0.21 \\
\hline - Mycophenolate mofetil & 35.3 & 3 & 0.002 \\
\hline - Mepolizumab & 0 & 24.2 & N/A \\
\hline - Azathioprine or methotrexate & 47.1 & 42.4 & 0.75 \\
\hline Relapse, n (\%) & $7(41.2)$ & $12(42.9)$ & 0.66 \\
\hline Exitus, n (\%) & $2(11.8)$ & $1(3)$ & 0.26 \\
\hline
\end{tabular}

* Med (IQR) for numerical data excluding age; mean \pm SD for ageANCA: Antineutrophil cytoplasmic antibody, BVAS: Birmingham Vasculitis Activity Score, ENT: Ear, nose, and throat IQR: Interquartile range, med: median, N/A: Not applicable, PNS: Peripheral nervous system, VDI: Vasculit damage index 


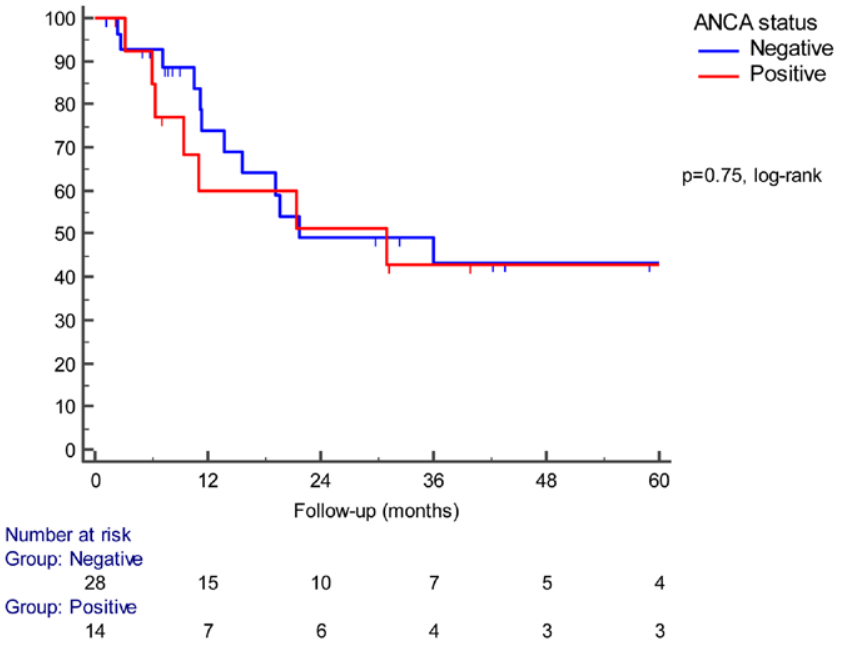

Figure 1. Kaplan-Meier relapse-free survival curve according to the ANCA status

Disclosure of Interests: None declared

DOI: 10.1136/annrheumdis-2021-eular.3261

\section{POS0252 IMPACT OF ANCA-ASSOCIATED VASCULITIS ON CLINICAL OUTCOMES AND RESOURCE UTILIZATION IN ELECTIVE PCI}

K. Mathias ${ }^{1}$, L. Mathias ${ }^{2}$, A. Mantha ${ }^{3}$, D. Arkfeld ${ }^{2} .{ }^{1}$ University of Chicago, Internal Medicine, Chicago, United States of America; ${ }^{2}$ USC Keck School of Medicine, Rheumatology, Los Angeles, United States of America; ${ }^{3}$ USC Keck School of Medicine, Internal Medicine, Los Angeles, United States of America

Background: Anti-neutrophil cytoplasmic antibody (ANCA) associated vasculitides (AV) can increase risk of atherosclerosis. ${ }^{1}$ Patients with autoimmune disease have increased rates of coronary events that are not fully explained by classic risk factors. ${ }^{2} \mathrm{~A}$ prior study has shown that autoimmune vasculitides -including polyarteritis nodosa, hypersensitivity granulomatosis with polyangiitis, giant cell arteritis, thrombotic angiitis, Takayasu arteritis, Goodpasture syndrome, and Kawasaki disease -are associated with increased cost of care and increased 30-day readmission after percutaneous coronary intervention (PCI). ${ }^{3}$ It is important to distinguish the cardiovascular outcomes of small-vessel ANCA-associated vasculitides from the other vasculitides included in this prior study because coronary arteritis is typically a complication of medium to large vessel vasculitis. Objectives: To investigate the impact of comorbid ANCA-associated small vessel vasculitis $(\mathrm{AV})$ on outcomes of $\mathrm{PCl}$ in a nationally representative cohort. Methods: Patients undergoing PCI in the 2016-2018 National Inpatient Sample were selected and those with ANCA-associated vasculitis were identified using the ICD-10 classification system. The NIS is an all-payer, survey-weighted, inpatient database estimating 37 million annual U.S. hospitalizations managed by the Healthcare Cost and Utilization Project (HCUP). Primary study outcomes were prevalence of comorbid vasculitis, mortality, complications, and cost of care.

Results: Of 1,351,670 patients undergoing PCl were identified, $410(0.03 \%)$ had diagnosis of ANCA-vasculitis. AV patients were similar to non-AV patients in distribution of gender, race, and median household income. AV patients were older $(70.9$ vs 65.2 years, $\mathrm{P}<0.001)$ and more likely to utilize Medicare $(78 \%$ vs $53 \%, \mathrm{P}<0.005)$. AV patients were more likely to present with non-ST segment myocardial infarction (NSTEMI) $(57.3 \%$ vs $41.5 \%, P<0.005)$ and less likely to present with ST-elevation myocardial infarction (NSTEMI) $(18.3 \%$ vs $31.3 \%$, $\mathrm{P}<0.05)$. AV patients had similar adjusted mortality $(6.1 \%$ vs $2.6 \%$, aOR: 2.1 , $P=0.8)$, cost of care $(\$ 32,985$ vs $\$ 25,767, \beta=0.02, P=0.7)$, and length of hospitalization (6.0 vs 3.9 , IRR: $1.2, \mathrm{P}=0.13$ ).

Conclusion: The presence of ANCA-associated vasculitis did not appear to negatively impact inpatient clinical outcomes or utilization of care among patients undergoing $\mathrm{PCl}$ in the United States. Cost of care was not increased, in contrast to an increase cost of care when small, medium, and large vasculitides are grouped together. The greater proportion of NSTEMI patients among those with AAV corresponds with a growing body of literature implicating inflammatory processes in the pathogenesis of certain forms of myocardial infarction meriting further investigation.

REFERENCES:

[1] Misra DP, Shenoy SN. Cardiac involvement in primary systemic vasculitis and potential drug therapies to reduce cardiovascular risk. Rheumatol Int. 2017;37(1):151-167. doi:10.1007/s00296-016-3435-1.
[2] Amaya-Amaya J, Sarmiento-Monroy JC, Rojas-Villarraga A. Cardiovascular Involvement in Autoimmune Diseases. El Rosario University Press; 2013. Accessed February 1, 2021. https://www.ncbi.nlm.nih.gov/books/ NBK459468/.

[3] Bailey KL, Mantha A, Sanaiha Y, Mathias L, Benharash P, Ebrahimi R. Short-Term Outcomes After Percutaneous Coronary Intervention in Patients With Autoimmune Vasculitides. J Am Coll Cardiol. 2019;74(1):159-160. doi:10.1016/j.jacc.2019.04.051.

Disclosure of Interests: None declared

DOI: 10.1136/annrheumdis-2021-eular.3369

\begin{tabular}{|l|l|}
\hline POS0253 & RITUXIMAB-BIOSIMILAR FOR ANTI-NEUTROPHIL \\
CYTOPLASMIC ANTIBODIES-ASSOCIATED \\
VASCULITIS: EXPERIENCE OF A SINGLE ITALIAN \\
CENTER
\end{tabular}

S. Sartorelli ${ }^{1}$, A. Cariddi ${ }^{1}$, L. Moroni ${ }^{1,2}$, E. Baldissera ${ }^{1}$, E. Bozzolo ${ }^{1}$, L. Dagna ${ }^{1,2}$. ${ }^{1}$ IRCCS San Raffaele Hospital, Unit of Immunology, Rheumatology, Allergy and Rare Diseases, Milan, Italy; ${ }^{2}$ Vita-Salute San Raffaele University, School of Medicine, Milan, Italy

Background: Rituximab (RTX), an anti-CD20 monoclonal antibody, represents a valuable treatment for anti-neutrophil cytoplasmic antibodies (ANCA)-associated vasculitis (AAV). RTX biosimilar CT-P10 (RTX-B) has been approved in Europe in all indications held by RTX originator (RTX-O). As stated by recent international consensus-based recommendations, ${ }^{1}$ there is evidence regarding safety and efficacy of biosimilars in the context of rheumatic diseases, but it is encouraged to gather additional data. ${ }^{2,3}$

Objectives: To report the experience of a single Italian center with RTX-B in AAV in terms of safety and efficacy.

Methods: We retrospectively reviewed the charts of all AAV patients followed up in our Small Vessel Vasculitis Clinic and we selected those who received RTX-B between October 2017 and May 2020, both naïve to RTX (RTX-Bn) or already treated with $\geq 1$ course of RTX-O and switched to RTX-B (RTX-Bs). Baseline features, disease outcome, concomitant therapy and adverse events 6 (T6), 12 (T12) and 24 (T24) months after RTX-B introduction, when available, were collected. Non-parametric statistic tests were used.

Results: Fifty-six AAV patients (44 [78.6\%] granulomatosis with polyangiitis (GPA), 12 [21.4\%)] microscopic polyangiitis (MPA)) received RTX-B with a median follow up of 20 (IQR 10-24) months. ANCA were positive in 49 (87.5\%) patients. Ten (17.9\%) patients were newly diagnosed with AAV, while $23(41.1 \%)$ had refractory disease and another 23 had relapsing disease.

Thirty-three (58.9\%) patients were RTX-Bs, whereas $23(41.1 \%)$ RTX-Bn. In $29(51.8 \%)$ patients RTX was decided because of remission induction, while in $27(48.2 \%)$ as maintenance regimen. Median cumulative RTX-B dose was 2.5 (2-3.875) grams with 3 (IQR 2-4) median courses. AAV activity, adverse events and concomitant therapy at T6, T12 and T24 are shown in Table 1. One GPA patient died of severe infection 3 months after a single infusion of RTX-B $500 \mathrm{mg}$ as maintenance therapy.

Considering the $52(92.6 \%)$ patients who reached a 6 months-follow up, we marked a significant decrease of number of patients on steroid therapy (39 [75\%] vs 27 [51.9\%]; $p=0.003$ ), of median prednisone daily dose (5 [IQR 5-25] vs 2.5 [IQR $1.25-5 \mathrm{mg} ; \mathrm{p}=0.001$ ) and of number of patient on concomitant bDMARDs (10 [19.2\%] vs $5[9.6 \%] ; p=0.003)$.

Analysing the $40(71.4 \%)$ patients who reached a 12 months-follow up, we confirmed the tendency with a decrease of number of patients on steroid therapy (29 [72.5\%] vs 13 [32.5\%]; $p=0.007)$, of median prednisone daily dose (5 [IQR $5-12.5]$ vs 3.75 [IQR 2.75-7.5]; $p=0.008$ ) and of number of patient on concomitant bDMARDs (9 [22.5\%] vs $3[7.5 \%] ; p=0.009)$.

No statistically significant difference was found for the same variables comparing T0 versus T24.

Table 1. AAV activity, concomitant therapy and adverse events at T0, T6, T12 and T24.

\begin{tabular}{lcccc}
\hline & T0 & T6 & T12 & T24 \\
\hline $\mathrm{n}(\%)$ & $56(100)$ & $52(92.6)$ & $40(71.4)$ & $18(32.1)$ \\
Dose RTX-B, median (IQR) grams & $1.75(0.5-2)$ & $0.5(0.5-1)$ & $0.5(0.5-1)$ & $0.5(0.5-1)$ \\
Concomitant prednisone therapy, n (\%) & $42(75)$ & $27(48.2)$ & $13(32.5)$ & $4(22.2)$ \\
Dose prednisone, median (IQR) & $5(5-15.625)$ & $2.5(2.5-5)$ & 3.75 & 2.75 \\
$\quad$ milligrams/day & & & $(2.75-7.5)$ & $(1.56-4.5)$ \\
Concomitant bDMARDs, n (\%) & $10(17.9)$ & $5(9.6)$ & $3(7.5)$ & $1(5.6)$ \\
Refractoriness & $\mathrm{NA}$ & $4(7.7)$ & $2(5)$ & $1(5.6)$ \\
Relapse & $\mathrm{NA}$ & $0(0)$ & $3(7.5)$ & $2(11.1)$ \\
Remission & $\mathrm{NA}$ & $48(92.3)$ & $35(87.5)$ & $15(83.3)$ \\
Suspension & $\mathrm{NA}$ & $10(19.2)$ & $9(22.5)$ & $4(22.2)$ \\
Infusion reaction, n (\%) & $1(1.8)$ & $1(1.9)$ & $0(0)$ & $0(0)$ \\
Infectious adverse events, n (\%) & $\mathrm{NA}$ & $10(19.2)$ & $6(15)$ & $3(16.7)$ \\
Severe infectious adverse events, n (\%) & $\mathrm{NA}$ & $2(3.8)$ & $1(2.5)$ & $0(0)$ \\
\hline
\end{tabular}

